\title{
A wireless crackmeters network for the analysis of rock falls at the Pietra di Bismantova natural heritage site (Northern Apennines, Italy)
}

\author{
A. Corsini, F. Bonacini, M. Deiana, R. Giusti, M. Russo \& F. Ronchetti \\ Università di Modena and Reggio Emilia, Dipartimento di Scienze Chimiche e Geologiche, Modena, Italy \\ C. Cantini \\ Comune di Castelnuovo ne' Monti, Ufficio Tecnico, Castelnuovo ne’ Monti, Italy
}

G. Truffelli

Regione Emilia Romagna, Servizio Tecnico Bacino Po, Parma, Italy

C. Iasio, M. Generali, L. Ascari, L. Chiesi \& L. Venturi

Henesis srl, Parma, Italy

\begin{abstract}
The Pietra di Bismantova (Northern Apennines, Italy) is a large calcareous sandstone rock slab which is distinctively bordered by sub-vertical cliffs that are affected by rock falls, a risk for people visiting the area and for key assets located at the foot of this natural heritage site. A wireless sensor network based on wireless nodes, crack meters and thermometers has been made operative in January 2015 in order to study the response of fractures to changing environmental conditions and support the spatial and temporal zonation of rock fall hazard in this natural heritage site. Results from the first eight months of monitoring show that intense snowfall and low temperatures can determine short-term pulses of fracture opening while the increase of temperature throughout summer determines long-term fracture closing trends. Moreover, as soon as February 2015 one of the crack meters monitored the rapid trend of crack opening that occurred prior to the failure of a large rock slab of about 200 cubic meters.
\end{abstract}

\section{INTRODUCTION}

The Pietra di Bismantova is a $400.000 \mathrm{~m}^{2}$ wide slab of calcareous sandstone overlaying marls and clay shales (Fig. 1). For its peculiar physical features and for its historical significance, it is one of the most important natural heritage sites in the National Park of the Tuscan-Emilia Apennine (Northern Apennines, Italy). It is distinctively bordered by sub-vertical lateral cliffs that are up to $100 \mathrm{~m}$ high (Fig. 1). Due to past tectonic stresses and for the effect of lateral spreading phenomena affecting the rock slab, the rock cliffs are pervasively affected by fractures that determine kinematic conditions for rock falls (Conti \& Tosatti 1994).

Rock falls have been a significant geomorphic factor in the area (GSUEG 1976). This is evidenced by the widespread rock falls deposits at the base of the cliffs, including rock blocks as large as more than thousand cubic meters.

Rock falls are also the main factor of natural risk for people visiting the area (tourists, hikers, climbers) and for buildings such as a small church (the so called "Eremo") and a restaurant located right at the base of the south-eastern cliff. Other authors have in the past analyzed and modelled possible rock fall trajectories in this site, concluding that the involvement of such assets is possible in case of rock failure and consequent rock falls (Migliazza \& Giani 2005; Borgatti \& Tosatti 2010).

Objective of this research is to investigate rock fall triggering conditions by analyzing the response of fractures opening to changing environmental conditions (precipitation and temperature). This can lead to the integration of spatial hazard zonation (based on rock fracturing patterns) with a temporal hazard zonation (based on the identification of critical meteorological conditions during which rock fractures opening evidences higher activity trends).

To this aim, an innovative Wireless Sensor Network (WSN) based on communication nodes and crack meters has been made operative in January 2015. The WSN monitors in pseudo-continue mode the behavior of several fractures bordering large and potentially unstable rock elements such as wedges, blocks, pillars and slabs. 


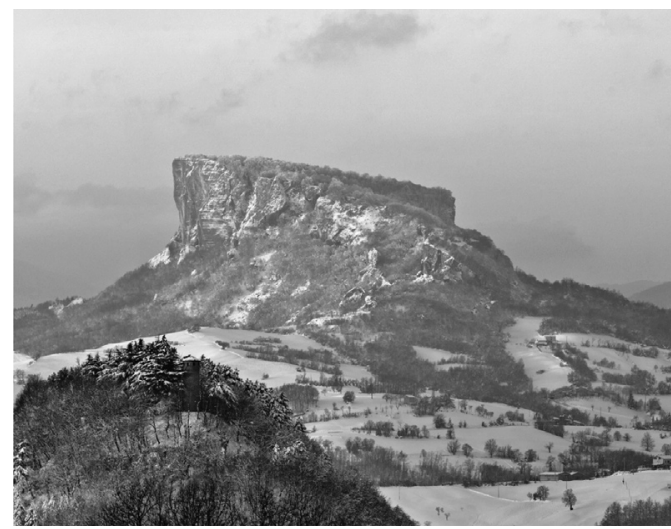

Figure 1. The Pietra di Bismantova (photo Bonacini F).

The south-eastern cliff has been selected as study site, because it is the area with the higher frequentation of people, it is the area at the base of which some of the largest pre-historic rock fall blocks can be found and, finally, because it is the area where, in recent times, quite large rock falls have occurred causing civil protection emergencies (specifically in $02 / 12 / 2012$ and in 13/02/2015, soon after the installation of the crackmeters network).

\section{MONITORING NETWORK}

\subsection{Wireless sensor network characteristics}

The monitoring system relies on a solar powered Wireless Sensor Network (WSN) based on a patented communication module. The installed system consists of 20 "nodes" installed in the cliff nearby rock fractures (Fig. 2). Each wireless node is equipped with four internal sensors (temperature, humidity, luminance and accelerometer for vibration detection), and an external wired uniaxial digital crack meter fixed across the joints that have to be monitored (Model Tecnopenta G1 with a travel distance of 25, 50 or $100 \mathrm{~mm}$, and an expected accuracy on the final measurement of $0.02 \mathrm{~mm}$ ). The 20 "nodes" send data to 1 "bridge" placed in a safety area at the bottom of the cliff. The "bridge" collects the data and sends them to the remote server by mean of GPRS connection. The data-storage system is based on redundant "cloud" servers hosted in California. To guarantee more security and accessibility in case of data recovery, the database is backed up on a local server hosted in Henesis' headquarter.

Each "node" samples its sensors every 5 minutes. The data collected are temporarily saved on-board, on a circular flash-memory, and transmitted to the "bridge", and from the "bridge" to the servers every 15 minutes. All these time intervals can be changed and customized for each sensor type by remote, through the web-based console.

The nodes have routing capability enabled and the proprietary WSN communication protocol has been ad-hoc developed by Henesis. For different applications, the Zigbee protocol can also be used with the same hardware. The protocol characteristics provide the WSN of self-configurable/selfhealing properties which continuously optimize the data transfer across the net towards the "bridge" according to "best-effort" criteria. Furthermore, the Henesis protocol enables bi-directional communication: 1) unicast from the nodes towards the server; 2) broadcast from the server towards the nodes (for remote software maintenance activities such as WSN reconfiguration, firmware updates, etc.).

The data are generally available for the customer via web-based interface, or "console", within twice the time-span set for the bridge-to-server communication from the acquisition. In the case of Bismantova where the bridge-server interval is set to 15 minutes, in case of direct communication nodebridge, it generally takes about 20-30 minutes from the data acquisition to its visualization. This time

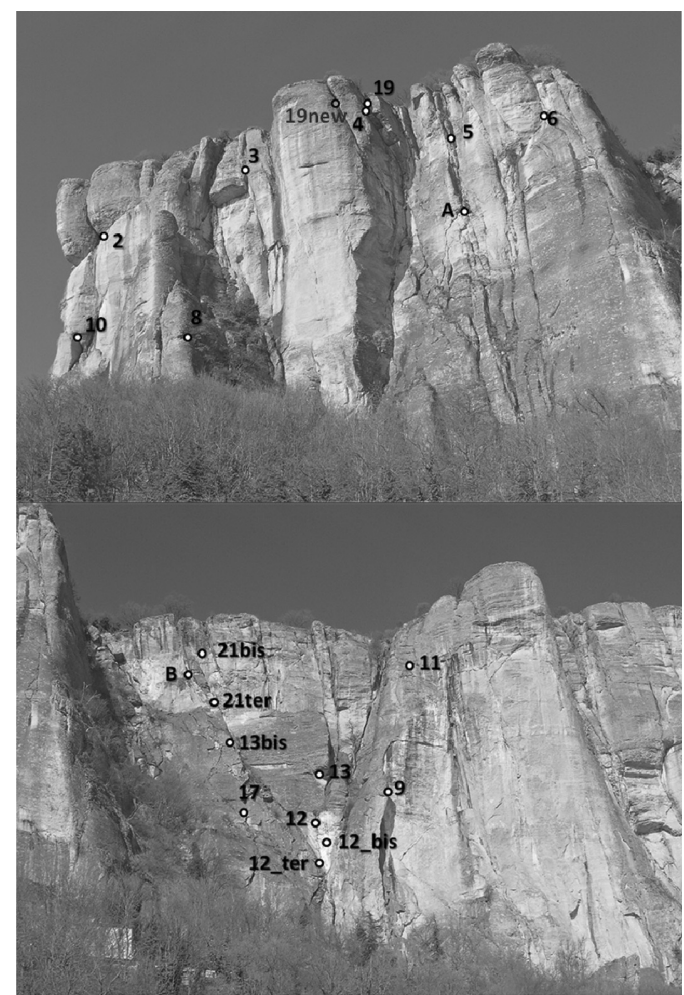

Figure 2. Location of WSN nodes equipped with crack meters. 
may enlarge depending on the number of jump (hop node-to node) the data has to carry out to reach the bridge.

The console can plot and compare several types of data. For this application the most significant analysis involved deformation measurement vs. temperature and humidity conditions.

\subsection{Field deployment}

The hardware customization and the field installations were designed for the specific environmental constrains, including the minimal on-site maintenance requirement, minimal visual impact as the monitoring site is a relevant touristic area, and reduced vulnerability from falling rocks. The system is entirely designed according to low (maintenance)cost and long-life criteria. The firmware is optimized for extremely low energy demand.

The enclosure satisfies IP67 requirements, and each node is furthermore protected by a small metallic roof. The antenna is placed beneath the node while the connection to the crackmeters is made through an ad-hoc tailored cable. Each node is fixed on the rock wall finding the best compromise between adequate exposure (for power and transmission requirements) and the local protection from rock falls. The external sensors are installed as close as possible within maximum $3 \mathrm{~m}$ of distance from the node (Fig. 3). The installation required a preliminary survey of each monitoring point and five days of rope work and climbing specialists, to setup the 20 monitoring point along the rock wall between 10 and $100 \mathrm{~m}$ of elevation from the ground.

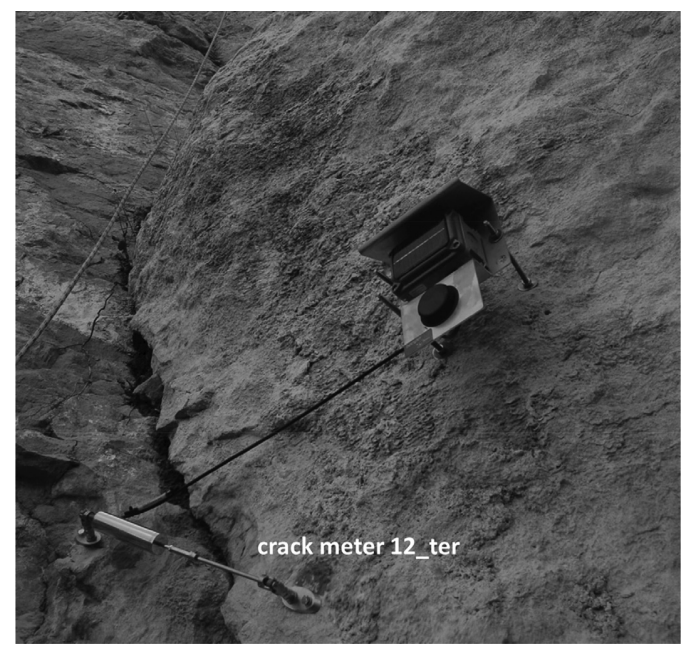

Figure 3. Example of field deployment of Beesper wireless nodes and crack meters.

\section{RESULTS}

\subsection{Overview of cracks opening variation trends}

In general, two quit distinct types of trends of rock fractures opening have been observed by crackmeters (Fig.4):

i. Trends that show a very limited temperature controlled cracks opening oscillation both on a daily basis (in the range of $0.1 \mathrm{~mm}$ ) and on a winter-to-summer seasonal basis ( $\max 0.5 \mathrm{~mm}$ ) (hereafter referred to as inactive trends);

ii. Trends that show a quite significant temperature controlled cracks opening oscillation both on a daily basis (in the range of $1 \mathrm{~mm}$ ) and on a winter-to-summer seasonal basis (max $5 \mathrm{~mm}$ ) (hereafter referred to as active trends).

Data show also that "active" or "inactive" trends should not be considered as indicative of incipient slope failure.

As a matter of fact, the trend of crackmeter 19, that was apparently inactive during the first month of monitoring, has then rapidly developed into an active trend in the time span of a few days, until failure occurred on 13th February 2015. This trend will be analysed in further detain in paragraph 3.2. On the other hands, in the few crackmeters that have shown an active trends since installation (such as the 12,12ter, 10, 9 and 19new) no failure has yet occurred. These trends will be analysed in further detain in paragraph 3.3.

\subsection{Cracks opening variations prior to failure}

In early February 2015, soon after a snowfall event that cumulated about 1 meter of snow in just a few

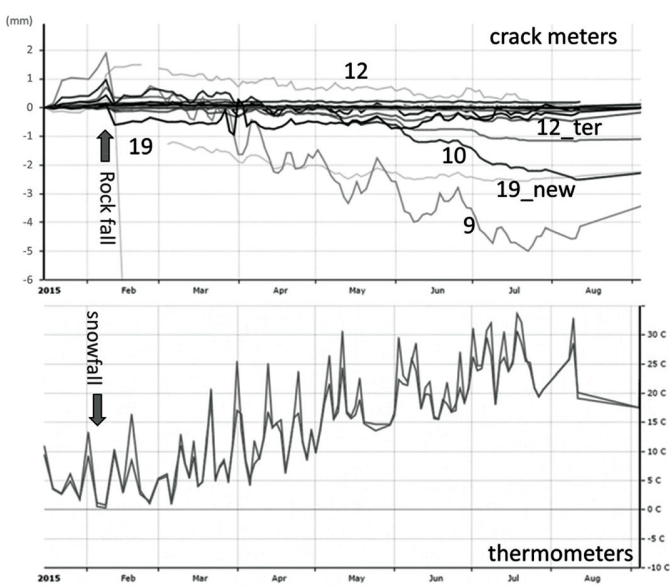

Figure 4. Trends of cracks opening variation vs. temperature. 
days, one of the monitored rock slabs underwent failure (Fig. 5a and Fig. 5b) due to the failure of the rock-bridge that was keeping it hinged upslope (Fig. 5b). This caused a block of about 200 cubic meter to fall for $100 \mathrm{~m}$ and to hit the small square next to the "Eremo" church at the base of the cliff (Fig. 5). Fortunately, only material damages occurred.

The crackmeter 19, installed in January 2015 in an open lateral crack (Fig. 5a) has captured the rapid evolution of crack opening prior to failure. More specifically, the cracks opening trend in the days leading to failure can be subdivided in 3 main phases (Figure 6).

During phase 1 , the crack was opening very slowly. The snowfall event that lasted from February 4th to February 7 th caused the trend to increase slightly. However, it remained of a very small magnitude, as a total opening of only about $0.2 \mathrm{~mm}$ was reached on February 7 th. In this period, temperature was progressively decreasing and remained below zero for almost all the time.

During phase 2, which begun on February 8th after the snowfall had ceased, a sudden rise in temperature occurred. The crack closed up of about $0.35 \mathrm{~mm}$ with minor oscillations related to daily thermal cycles. In practice, from February 9th onward the temperature never reached below zero,
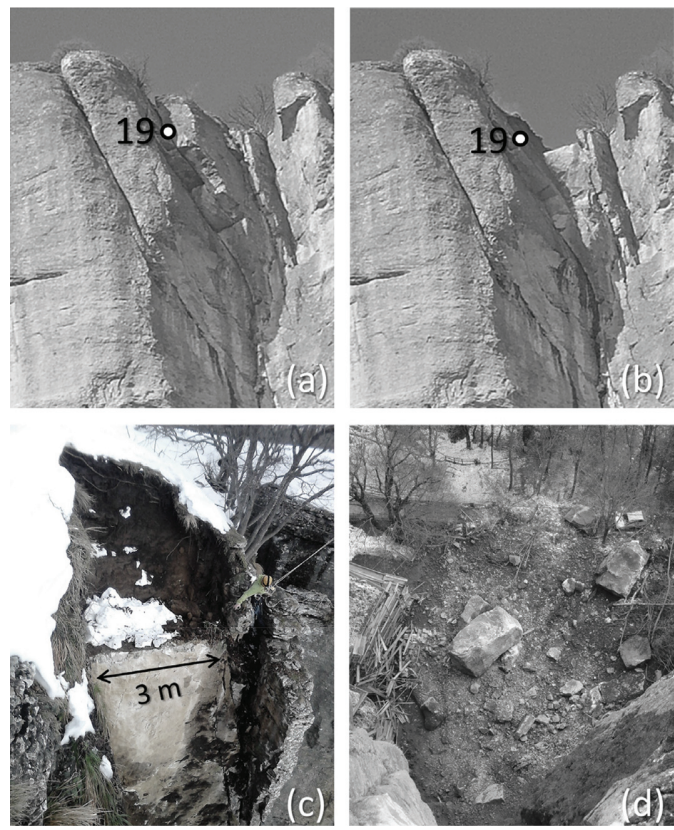

Figure 5. (a, b) Location of crackmeter 19 prior and after the February 13th 2015 rock fall. (c) new fracture behind the fallen block. (d) rock fall deposits near the church.
Table 1. Crack opening progression during phase 3 leading to the rock fall of February 13th 2015.

\begin{tabular}{lll}
\hline Date-Time & Value $(\mathrm{mm})$ & Increment $(\mathrm{mm})$ \\
\hline 12/02/15-21:00 & $7.70 \mathrm{~mm}$ & (base value) \\
13/02/15-11:00 & $8,11 \mathrm{~mm}$ & $+0,41$ \\
$13 / 02 / 15-12: 00$ & $8,47 \mathrm{~mm}$ & $+0,77$ \\
$13 / 02 / 15-12: 30$ & $9.29 \mathrm{~mm}$ & +1.59 \\
$13 / 02 / 15-12: 40$ & $13.33 \mathrm{~mm}$ & $+5,64$ \\
$13 / 02 / 15-12: 50$ & out of range & (rock fall) \\
\hline
\end{tabular}
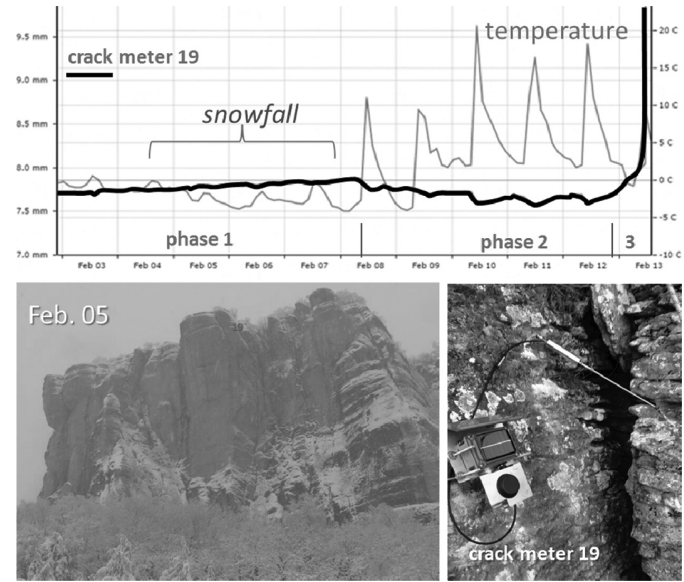

Figure 6. (up) Plot of cracks opening progression prior to the rock fall of February 13th 2015. (lower left) picture of the slope of February 5th showing accumulation of snow also on the vertical cliffs. (lower right) picture of crackmeter 19 .

even at night, but the difference between night and midday temperature was as high as $20^{\circ} \mathrm{C}$.

During phase 3, which begun the evening of February 12 th, the temperature dropped again below zero and a progressive opening trend started. The crack opening progression that led to rock failure is roughly exponential (Fig. 6). The "base value" of $7.7 \mathrm{~mm}$ reached in phase 1 was reached at 21:00 of February 12th. (Tab. 1). An incremental opening exceeding $1 \mathrm{~mm}$ from the base value was reached at 12:30 on February 13th. Failure occurred between 12:40 and 12:50 on February 13th. This rapid progression indicates how critical it would be to issue early warnings with an adequate advance.

\subsection{Cracks opening variations without rock failure}

The crackmeters that have shown active trends since installation are: 12, 12ter, 10 and 9. These fractures showed a sudden opening (as high as $2 \mathrm{~mm}$ ) in early February 2015, due to the rapid drop of temperature and the snowfall event 


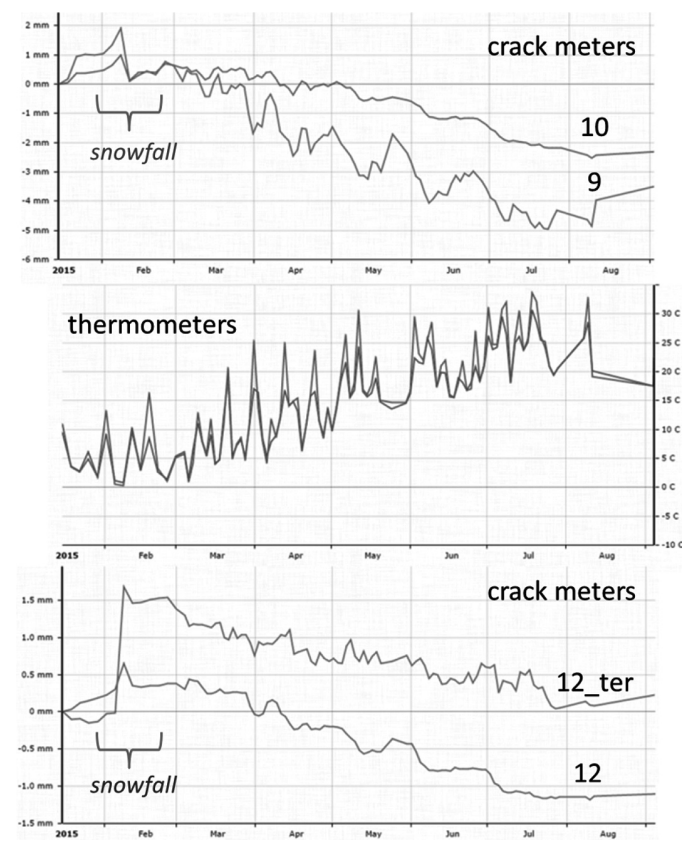

Figure 7. Cracks opening plots of crackmeters showing active trends in comparison to temperature variation.
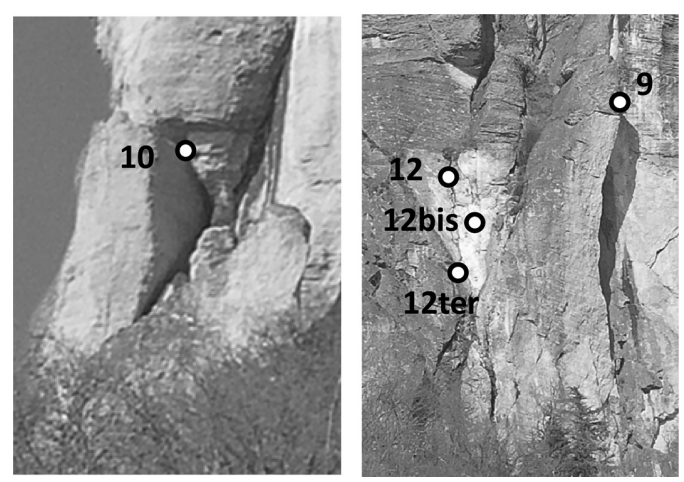

Figure 8. Location of crackmeters showing active trends. Crackmeters 9 and 10 monitor the back crack of large rock pillars, whereas 12,12 bis and 12 ter monitor fractures of rock blocks on the area of the $02 / 12 / 2012$ rock fall event.

(Fig. 7). After that, they all showed a progressive closure that has continued throughout the spring and summer 2015. This behaviour is most likely related to the seasonal increase of temperature.

The seasonal trend has accounted for up to $5 \mathrm{~mm}$ closure in the case of crackmeters 10 and 9 that monitor the back-crack of very large rock pillars (Fig.8). On the other hand, the seasonal trend has been limited to a closure in the order of $1 \mathrm{~mm}$ in crackmeters 12 and 12ter, that monitor the lateral crack of large rock blocks in the area were the rock fall event of 02/12/2012 occurred (Fig. 8). Daily or weekly oscillations due to temperatures are also quite clearly visible, and are of an higher magnitude in crackmeters monitoring the large rock pillars that, evidently, have a tower-like oscillating behaviour.

\section{DISCUSSION AND CONCLUSION}

Result from the first eight months of crackmeters monitoring using an innovative WSN shown that this type of information can be of great support on the study of the conditions leading to rock falls.

Specifically, intense snowfall and low temperatures have determined short-term pulses of fracture opening while the increase of temperature throughout summer has determined long-term fracture closing trends. Moreover, intense snowfall with accumulation on the cliff and the subsequent drastic change of temperature regime (leading to rapid snow melting and, possibly, to cycles of freeze-thaw into fractures) are probably among the causes that progressively led to the rock fall event of February 13th 2015. Cracks opening during the snowfall period indicates that ice formed in the main fracture inducing pressure. This has probably caused stress in in the rock bridge that - prior to failure - kept the whole block attached to the slope (depicted after failure in the photo of Fig. 5a). When snowmelt started the crack rapidly closed up. But, finally, the night before failure, temperature dropped once again below zero. Once again, cracks opening occurred as ice probably formed in the fracture, since. This induced an additional cycle of stress in the rock bridge, determining its failure and, consequently, the rock fall.

It should be pinpointed that the rock fall event was preceded by a trend of crack opening that became very rapid only a few tens of minutes prior to failure. This evolution indicates that an early warning simply based on cracks opening thresholds would be very critical, as the rapidity at which cracks opening develops prior to failure did not allow, on realistic terms, to assure the enough warning time to carry out the consequent evacuation actions. Nevertheless, the high temporal sampling rate of crackmeters in this WSN is fundamental for an effective analysis of the involved variables and to understand the processes and their response to changing weather. In perspective, when longer time series of data will be analyzed, the identification of critical weather conditions can lead to a temporal zonation of hazard, i.e. to the identification of periods during which the access to some parts of the area might be limited (similar to what is generally done for snow avalanches hazard). This temporal approach to hazard assessment, coupled with a spatial zonation of susceptible zones, 
is believed capable of enhancing risk prevention policies in this site. Moreover, thanks to the high frequency of monitoring, a warning system can be based on the identification of "anomalies" with respect to specific mid to long term trends rather than threshold values only.

\section{ACKNOWLEDGEMENTS}

This work is carried out within Research project "definition of a geological technical knowledge framework about the conditions of instability of the Pietra di Bismantova" supported by the Municipality of Castelnovo ne' Monti. The installation of the monitoring network was supported by specific funds of the Civil Protection Agency of the Emilia-Romagna Region.

\section{REFERENCES}

Borgatti, L. \& Tosatti, G. 2010. Slope instability processes affecting the Pietra di Bismantova Geosite (Northern Apennines, Italy). Geoheritage 2(3): 155-168.

Conti, S. \& Tosatti, G. 1994. Caratteristiche geologicostrutturali della Pietra di Bismantova e fenomeni franosi connesssi (Appennino reggiano). Quaderni di Geologia Applicata 1: 25-43.

GSUE (Gruppo di Studio Università Emiliane per la geomorfologia (1976). Geomorfologia dell'area circostante la Pietra di Bismantova (Appennino reggiano). Boll. Serv. Geol. d'Italia 97, Roma.

Migliazza, M. \& Giani, G.P. (2005) Analisi di fenomeni di crollo nella Pietra di Bismantova (RE). GEAM. Geoingegneria Ambientale e Mineraria 42(116): 41-50. 